\title{
Wireless logging of extracellular neuronal activity in the telencephalon of free-swimming salmonids
}

\author{
Susumu Takahashi ${ }^{{ }^{*}}$ (D), Takumi Hombe ${ }^{2}$, Riku Takahashi ${ }^{1}$, Kaoru Ide ${ }^{1}$, Shinichiro Okamoto ${ }^{3}$, Ken Yoda ${ }^{2}$, \\ Takashi Kitagawa ${ }^{4}$ and Yuya Makiguchi ${ }^{5^{*}}$
}

\begin{abstract}
Background: Salmonids return to the river where they were born in a phenomenon known as mother-river migration. The underpinning of migration has been extensively examined, particularly regarding the behavioral correlations of external environmental cues such as the scent of the mother-river and geomagnetic compass. However, neuronal underpinning remains elusive, as there have been no biologging techniques suited to monitor neuronal activity in the brain of large free-swimming fish. In this study, we developed a wireless biologging system to record extracellular neuronal activity in the brains of free-swimming salmonids.
\end{abstract}

Results: Using this system, we recorded multiple neuronal activities from the telencephalon of trout swimming in a rectangular water tank. As proof of principle, we examined the activity statistics for extracellular spike waveforms and timing. We found cells firing maximally in response to a specific head direction, similar to the head direction cells found in the rodent brain. The results of our study suggest that the recorded signals originate from neurons.

Conclusions: We anticipate that our biologging system will facilitate a more detailed investigation into the neural underpinning of fish movement using internally generated information, including responses to external cues.

Keywords: Fish biotelemetry, Extracellular neuronal recording, Salmonids

\section{Background}

Some fish, birds, and mammals exhibit outstanding navigational abilities, such as mother-river homing, seemingly possessing cognitive maps. Current self-location and compass bearings are required for such spatial navigation. In mammals, extracellular electrophysiology for freely navigating animals has led to the discovery of a variety of space-responsive cells, including place cells [1] and grid cells [2], which fire maximally at specific

\footnotetext{
*Correspondence: stakahas@mail.doshisha.ac.jp; yuya.makiguchi@gmail.com ${ }^{1}$ Laboratory of Cognitive and Behavioral Neuroscience, Graduate School of Brain Science, Doshisha University, Kyotanabe City, Kyoto 610-0394, Japan

${ }^{5}$ College of Bioresource Sciences, Nihon University, Kanagawa 252-0813, Japan

Full list of author information is available at the end of the article
}

locations, and cells that fire maximally in response to specific heading directions [3]. These internally generated maps and compasses are thought to contribute to spatial navigation [4].

Until recently, there have been few reports of spaceresponsive cells in fish brains. This lack of information is mainly due to the difficulties of underwater neuronal recording. Zebrafish, a small, laboratory-bred, and nonmigrant fish widely used as an animal model in neuroscience, can be genetically modified to record neuronal activity through optical imaging of intracellular calcium dynamics. However, head clamping and genetic engineering are prerequisites for such neuronal recordings. While state-of-the-art technology has enabled us to image neuronal activity in the brains of free-swimming 
zebrafish, they must be kept in a small restricted area under a microscope [5].

Therefore, the neural underpinning of fish movement cannot be examined in the field using the aforementioned methods. For medium-sized fish, tethering cables are a prerequisite for recording extracellular neuronal activity from the brain; however, the apparatus generates significant torque in the water, severely inhibiting voluntary movement. Despite such restrictive limitations, there have been reports of the presence of space-responsive cells in the brains of non-migrant fish [6].

Recent remarkable advances in microelectromechanical systems have led to the rapid evolution of biologging techniques. For instance, a lightweight logger for extracellular neuronal activity, called a neurologger, helped to discover place cells and head direction cells in the brains of flying bats [7]. Furthermore, a neurologger stored in a waterproof case [8] led to the discovery of neurons in the telencephalon of goldfish (Carassius auratus), whose activity was found to be correlated with swimming speed and a specific heading vector as they swam along a wall [9].

Theoretically, this method, pioneered on goldfish, could be applied to larger fish weighing several kilograms. However, the electrode implantation procedure developed for medium-sized goldfish is not suitable for larger fish, as their brains are located deeper beneath the head surface. For example, the trout brain is located 3-4 cm from the head surface, whereas the goldfish brain lies only a few millimeters underneath [9]. In addition, powerful (weight: approximately $2 \mathrm{~kg}$ trout vs. 100 g goldfish) and fast (average speed of approximately $20 \mathrm{~cm} / \mathrm{s}$ ) swimming may affect logger protection and electrode stabilization.

In the present study, combining the pioneering wireless logging study developed in goldfish [8] with our extracellular recording techniques for rodents $[10,11]$, we developed a system for recording space-responsive cells in the brains of large migrant fish. To verify the system validity, we recorded multiple neuronal activities from the brains of trout swimming freely in a rectangular tank. Then, we examined the correlations between swimming behaviors and single neuron firing rates.

\section{Methods}

\section{Biotelemetry system experimental setup}

We developed a wireless biologging system that enables the monitoring of extracellular neuronal signals from the brains of free-swimming trout. Figure 1a shows the experimental setup for the simultaneous monitoring of neuronal activity and swimming trajectory. We used an array of extracellular tetrodes to record extracellular neural activities. This device is an electrode consisting

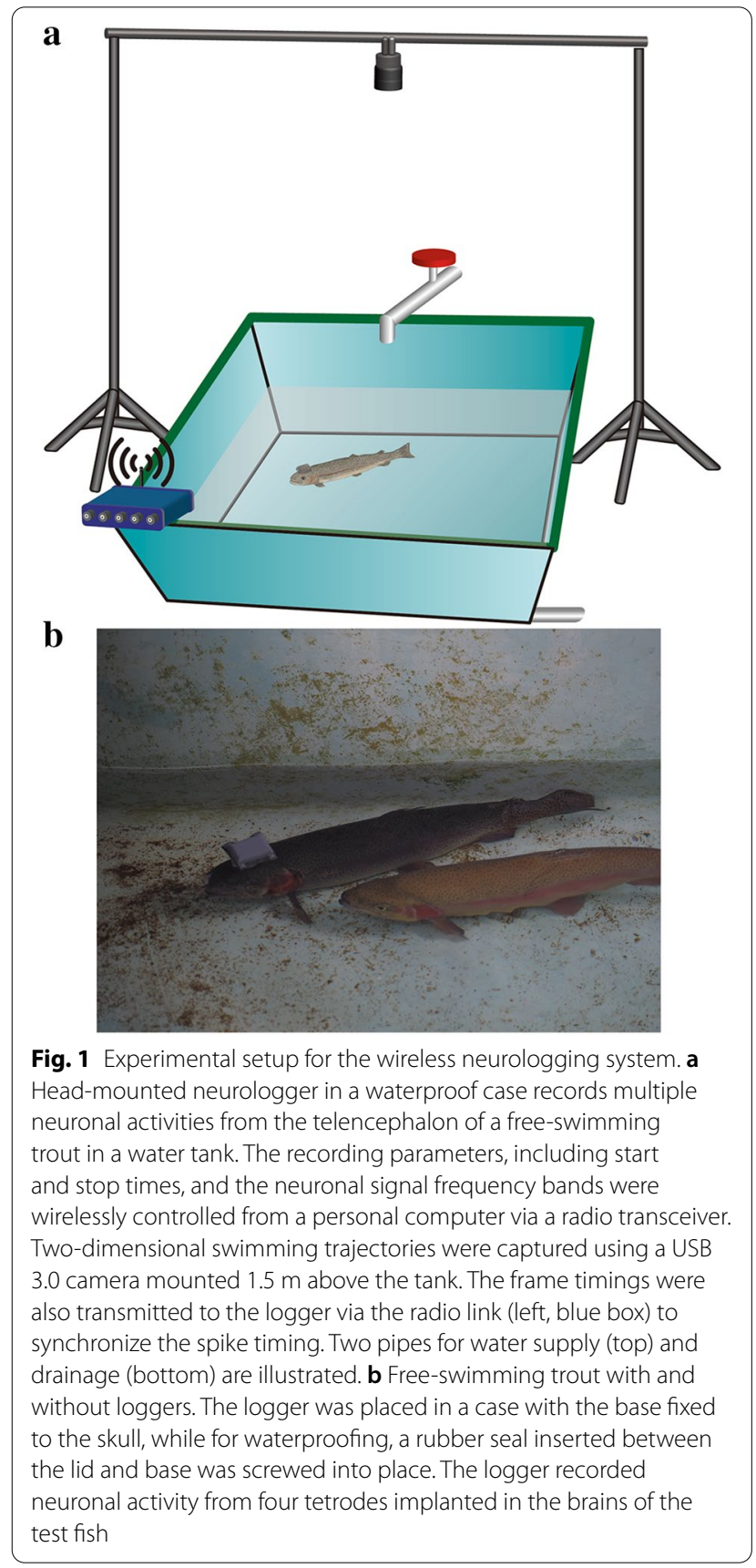

of four twisted microwires; it has become a standard electrode widely used to simultaneously record multiple single-neuronal activities in rodent brains [12]. Extracellular neuronal signals were stored on an SD card using a commercially available neurologger (Mouselog-16B, Deuteron Technologies, Jerusalem, Israel) placed in a custom-made, 3D-printed, waterproof case (Fig. 1b). Using dedicated control software for MouseLog-16, we controlled the neurologger via a transceiver that transmitted radio signals on the $915-\mathrm{MHz}$ frequency band. 
The neurologger also recorded external trigger signals to identify where the fish was located in the water tank when the action potential occurred from the neuron. These signals were recorded for each frame captured by a USB 3.0 video camera (ace acA3088-57uc, Basler AG, Ahrensburg, Germany) mounted $1.5 \mathrm{~m}$ above the water tank and transmitted from a personal computer via a radio link. As was the case in the previous study, the radio link was available within a few tens of centimeters below the water surface. The details are described in the following subsections.

\section{Animals}

The three fish (rainbow trout [Oncorhynchus mykiss]; body weights: 1,548-2,230 g) used in the experiment were purchased from the Fuji Trout farm in Fujinomiya City, Shizuoka Prefecture, Japan, where they were fed commercial pellets once a day. To facilitate the neurologger attachment, we conducted our experiments at the Fuji Nature Education Center of Nihon University. We chose our experimental fish without discriminating for sex. After purchase, they were transported by car to the Education Center, where they were acclimated in spring water at approximately $9{ }^{\circ} \mathrm{C}$ until required for experimentation.

The fish were not fed during the experiment, and all procedures were approved by the University of Tokyo Institutional Animal Care and Use Committee.

\section{Surgery}

The experimental fish were immersed in an anesthetic solution, prepared by adjusting FA 100 (eugenol; Tanabe Seiyaku Co. Ltd, Osaka, Japan) to a concentration of $0.5 \mathrm{~mL} / \mathrm{L}$, for 5-10 min, until their gill lids ceased moving. When the experimental fish were immobilized, they were fixed to an acrylic apparatus on a specific mounting table to facilitate the electrode implantation and neurologger attachment (Fig. 2a). The anesthesia, prepared with FA 100 at a concentration of $0.25 \mathrm{~mL} / \mathrm{L}$, was refluxed from the mouth to the gills by tube, using a peristaltic pump for circulation. The fish back was covered with a towel, kept wet during the experiment by pumping water onto it to prevent the body from drying. During the experiment, a bag of ice was added to the water tank every $30 \mathrm{~min}$ to prevent sudden increases in water temperature, maintaining it at approximately $9^{\circ} \mathrm{C}$.

With the help of an imaginary line connecting the back of the eyes as a starting point, a rectangular portion of the epidermis was removed with a scalpel for a length of 3-4 cm toward the caudal fin. A micro-drill was then used to prepare an oval hole in the exposed skull, followed by a meticulous procedure intended to avoid damage to the brain. After removing the excised skull, fat and tissue fluid were gently extracted with a Kimwipe (Kimtech ${ }^{\circledR}$ Science $^{\mathrm{TM}}$ Kimwipes $^{\mathrm{TM}}$ ) to expose the olfactory bulb, telencephalon, and optic tectum.

For the anchor screw, a maximum of 12 holes equally spaced were drilled along the edge of the oval hole. The number of holes was determined on a subject-bysubject basis to avoid scattered cartilaginous tissues that mainly reside in the rostral area. After the anchor was screwed into the holes, the sites were covered with dental acrylic resin (Unifast III, GC Inc., Japan). The ground wire was implanted into the medial optic tectum. The tetrode array described below was implanted into the targeted recording locations of the telencephalon with micromanipulators (SM-15L or SMM-200, Narishige Inc., Japan, respectively) (Fig. 2b). Since the brain depth was approximately $3-4 \mathrm{~cm}$ below the skin, the precise coordination of electrode locations was performed on a case-by-case basis.

The space above the brain was filled with Vaseline, while the space between the head and guide tubes was filled with small, 3D-printed pieces and then covered with dental acrylic resin. Finally, the neurologger case was attached to the electrodes, fixed, and covered with dental acrylic resin (Fig. 2c). All operations were completed within $90 \mathrm{~min}$. After surgery, the fish were immediately moved to an outdoor experimental tank $(145 \mathrm{~cm} \times 105 \mathrm{~cm})$ and acclimated until they were observed to swim normally.

\section{Electrode fabrication}

Based on the experience acquired in rodent studies $[10,11]$, we used tetrodes containing four tungsten microwires (12.5 $\mu \mathrm{m}$, HML-coated, California Fine Wire, CA, USA), twisted and bundled with a heat gun, to record extracellular neuronal activity. Each tetrode was inserted into a polyimide tube (inner diameter: $0.04 \mathrm{~mm}$, outer diameter: $0.20 \mathrm{~mm}$ ). The tube array was constructed by gluing the tubes in a concentric circle arrangement, with a distance of approximately $0.2 \mathrm{~mm}$ between them (Fig. 3a). To stabilize the electrode drift, a flexible electrode tip (approximately $0.5 \mathrm{~cm}$ ) was exposed from the tubes to absorb the torque caused by the trout's powerful and fast swimming. Each microwire within a tetrode was crimped to the corresponding hole in the electrode interface board (EIB) using a gold pin (Fig. 3b, c). Immediately before surgery, the tip of the tetrode was cut at a right angle. The impedance was approximately $600 \mathrm{k} \Omega$ at a frequency of $1 \mathrm{kHz}$. A ground electrode, an insulated stainless-steel wire (diameter: $0.2 \mathrm{~mm}$ ) implanted into the medial optic tectum, also served as a reference point for neuronal recordings (Fig. 3c, green wire). 
a

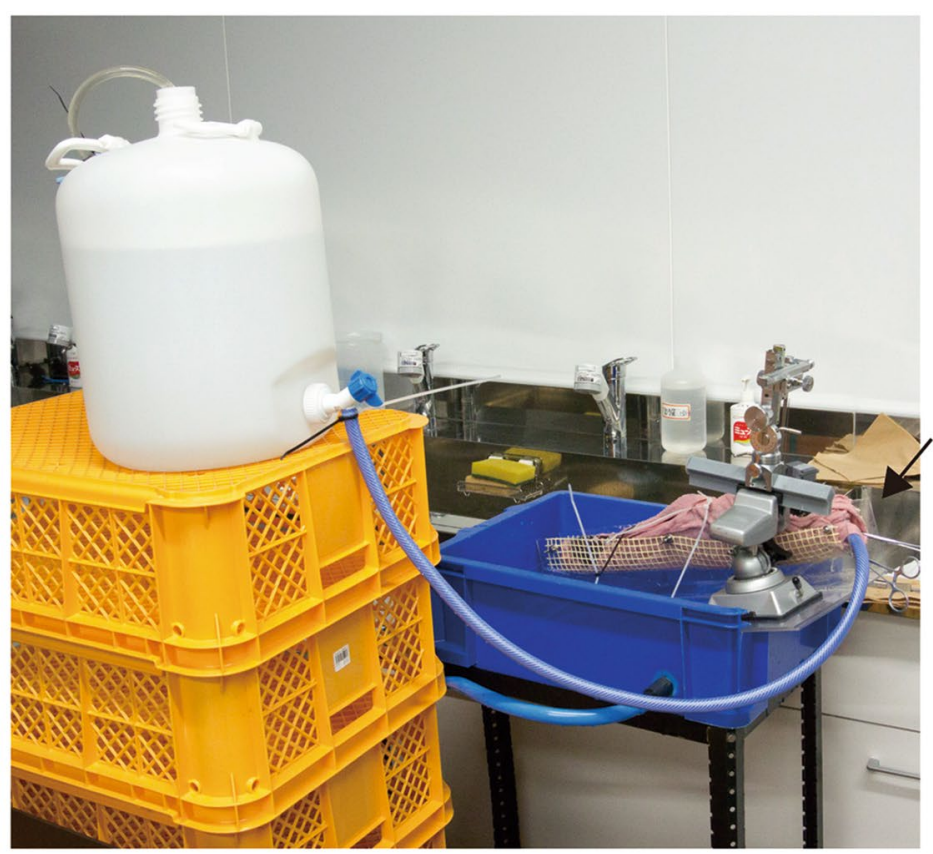

b

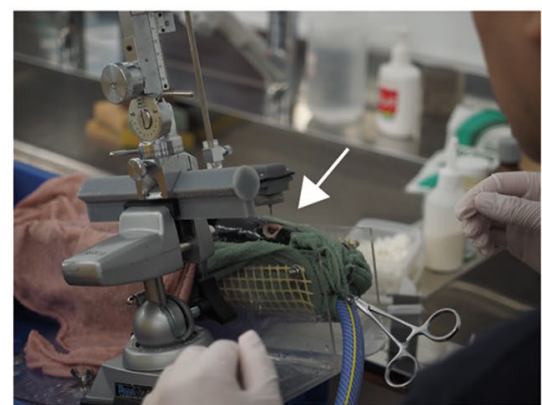

c

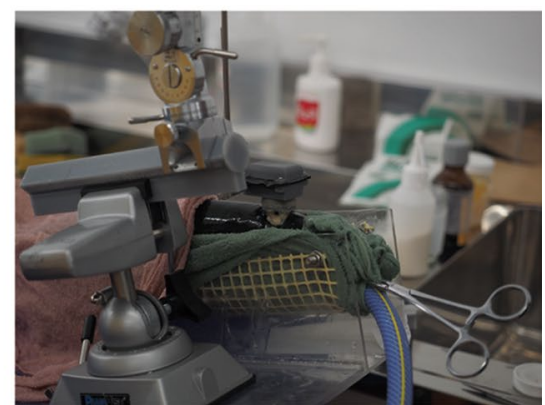

Fig. 2 Surgery for tetrode implantation and case fixation. a To implant the tetrodes into the brain, each anesthetized fish was fixed to an acrylic fixture on a custom-made mounting table (arrow). The anesthetic solution was circulated using a peristaltic pump. The fish back was covered with a wet towel (pink) to prevent the body from drying, and water was poured onto it with a small pump during the experiments. A bag of ice was added to the water tank every 30 min during the experiments to prevent sudden water temperature increases. $\mathbf{b}$ Oval hole drilled into the exposed fish skull (white arrow). A scalpel was used to excise a rectangle of epidermis in the direction of the caudal fin, starting from an imaginary guideline between each fish's eyes. To expose the olfactory bulb, the telencephalon, optic tectum, fat, and tissue fluid were carefully removed. Anchors were screwed into holes equally spaced along the edge of the oval hole and covered with dental acrylic resin. c A ground wire and tetrode array were implanted into the medial optic tectum and target recording locations of the telencephalon using micromanipulators. The space above the brain was then filled with Vaseline, while the space between the head and electrode interface board (EIB) was filled with small, 3D-printed pieces and then covered with a dental acrylic resin. The neurologger case was attached to the EIB and covered with dental acrylic resin

(See figure on next page.)

Fig. 3 Electrode array and waterproof case for neurologger. a An array of tetrodes; each tetrode was inserted into a polyimide tube, and the tubes were glued together to construct an array with constant internal spacing. $\mathbf{b}$ Housing for connecting the tetrode array to the custom-made electrode interface board (EIB). c Each tetrode microwire was crimped into a corresponding contact hole using a gold pin. $\mathbf{d}$ Cover to attach the EIB to the tetrode array. $\mathbf{e}$ Tetrode array assembly. $\mathbf{f}-\mathbf{g}$ Tetrode array with an undercover to connect to the logger case (f). $\mathbf{h}-\mathbf{j}$ Base (h), support (i), and lid (j) for the custom-made, 3D-printed, waterproof case. $\mathbf{k}$ Lid with rubber seal. I The base was attached to the housing and covered with dental acrylic resin (scale bar $=1 \mathrm{~cm}$ ) 


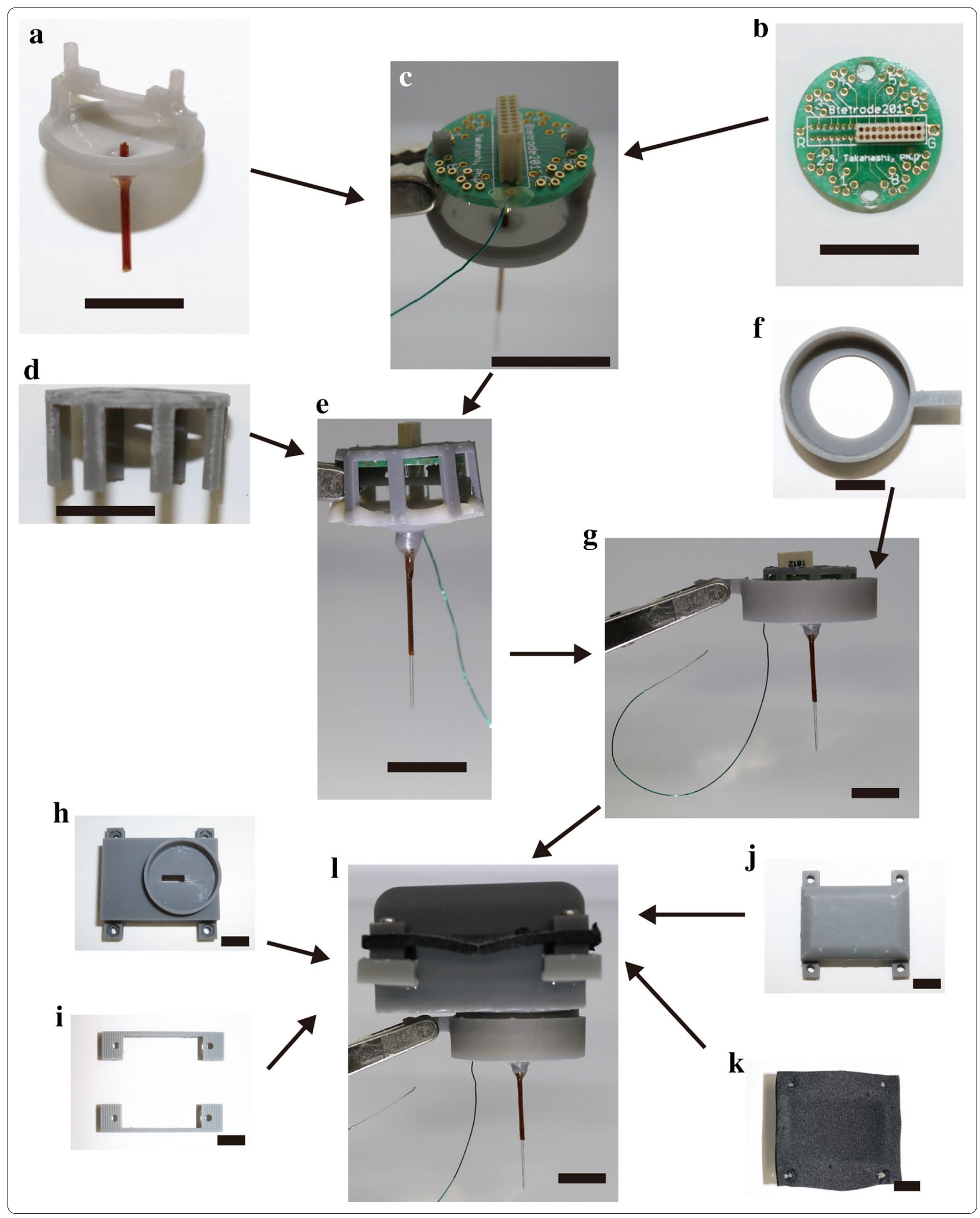


a

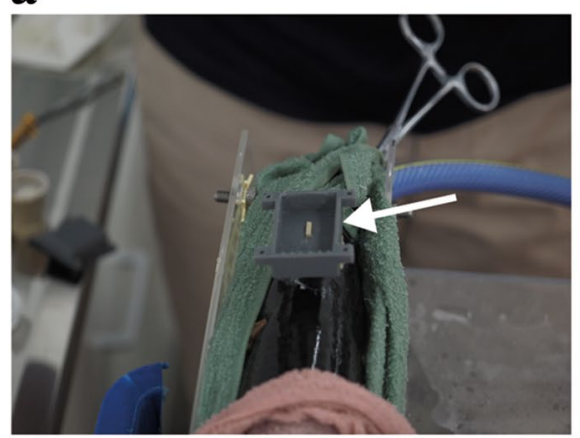

c

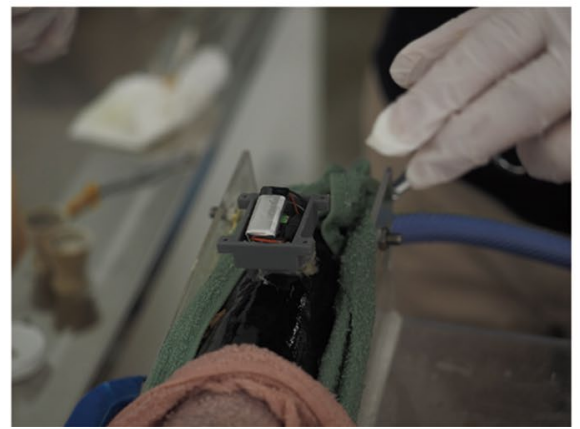

b

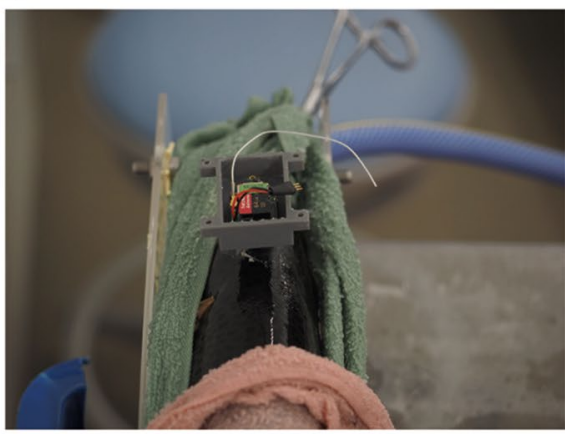

d

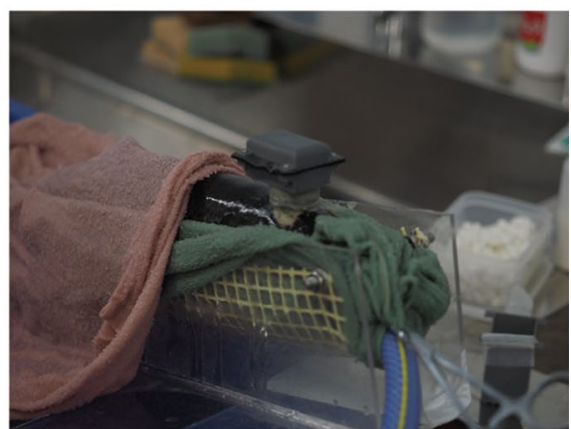

Fig. 4 Attaching a logger. a To prevent water ingress, the slight gap between the base and the socket of the neurologger (white arrow) was filled with an ultraviolet-curable adhesive immediately after the electrode was implanted. b-c Each day, before the initial recording session, the neurologger, including its SD card (b) and battery (c), was placed in the waterproof case, while the anesthetized fish was fixed to the mounting table. $\mathbf{d}$ After the neurologger and the battery were attached to the base, the lid, complete with its rubber seal, was screwed into the base using four screws

\section{Waterproof case}

We manufactured a waterproof neurologger case using a pioneering study on goldfish as a reference [8]. The base, lid, and tetrode array housing were fabricated using a $3 \mathrm{D}$ printer (Fig. 3h, j) (Form 2 or 3, Formlabs, MA, USA). The base and tetrode array housing were designed to mesh with each other, and the tetrode array was connected to the neurologger through a hole in the base. The slight gap between the base and the socket was filled with an ultraviolet-curable adhesive (Bondic $^{\circledR}$, Bondic Co., NY, USA) (Fig. 4a, arrow). After the base and housing were covered with dental acrylic resin, the lid with a rubber seal (chloroprene, thickness: $2 \mathrm{~mm}$ ) (Fig. 3k) was placed on the base and tightened using four screws (Figs. 3l, 4d). For logger protection against high torque, we added a lid, in addition to the base and supports, to close the case. The outer dimensions of the waterproof case were $5 \mathrm{~cm} \times 5 \mathrm{~cm} \times 3 \mathrm{~cm}$. The total weight of the case was approximately $17 \mathrm{~g}$. Before the initial recording session, the neurologger and battery were placed in the waterproof case, and the anesthetized fish was fixed to the mounting table (Fig. 4b, c).

\section{Neurologger}

Before the extracellular neuronal signals were stored in an SD card, they were unity-gain buffered, digitized, and continuously sampled at $31.25 \mathrm{kHz}$ with an RHA2000 chip (Intan Technologies, CA, USA) in the MouseLog$16 \mathrm{~B}$, operating in either wide- $(1-7000 \mathrm{~Hz})$ or narrowband $(300-7000 \mathrm{~Hz})$ mode. We simultaneously recorded up to four tetrodes (16 channels) for over one hour with a battery $(3.7 \mathrm{~V}, 170 \mathrm{mAh})$. The weight of the neurologger, including the battery, was approximately $6 \mathrm{~g}$. We used a magnetic switch to turn on the neurologger immediately before each recording session to reduce battery consumption.

\section{Offline preprocessing}

After the neuronal signals were downloaded from the SD card, action potential (spike) data were digitally filtered at $800-7500 \mathrm{~Hz}$. The tetrode recording contained multiple single-neuronal activities. The spikes were isolated using spike-sorting software (KlustaKwik, open-source software by Harris Lab, UCL, London, UK, and available from https://sourceforge.net/projects/klustakwik) and manually verified [13] to extract individual neuronal 
$\mathbf{a}$

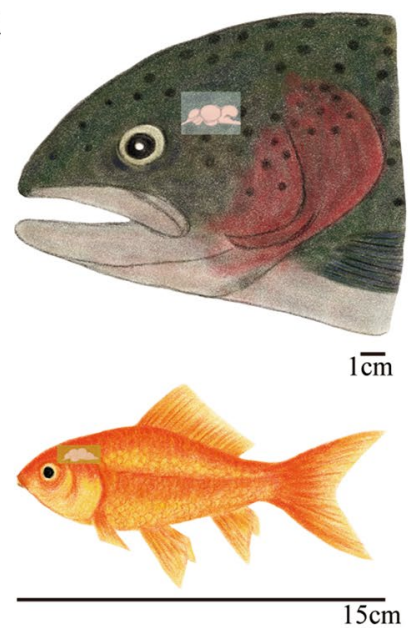

c

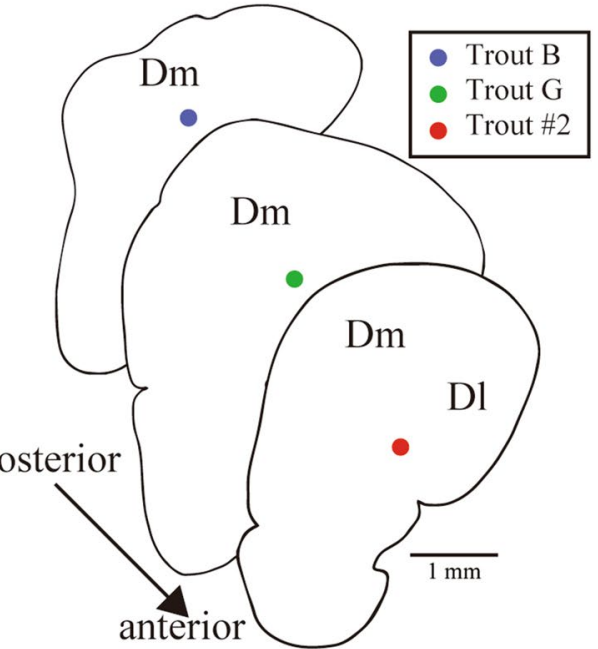

b

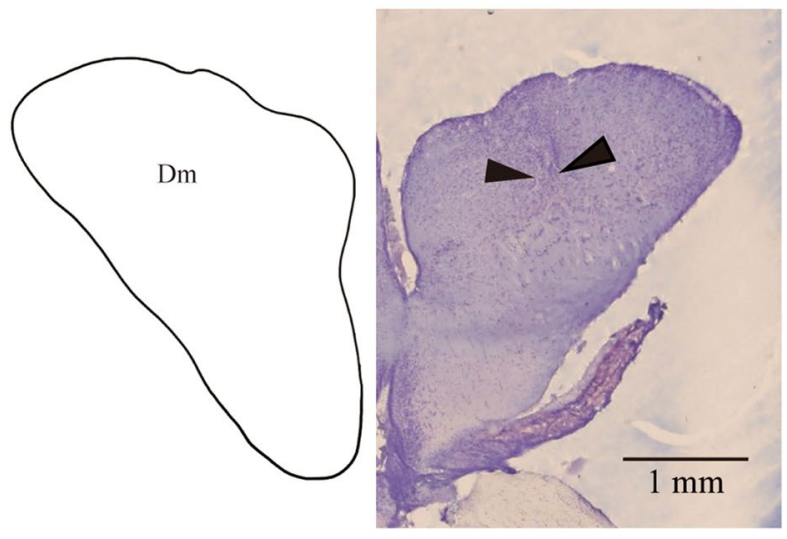

d

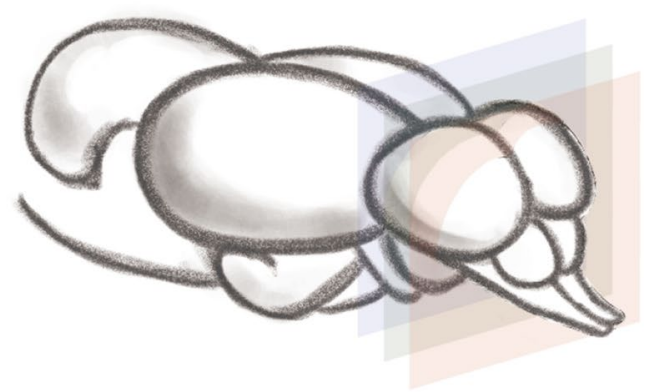

Fig. 5 Histological identification of recording locations in the brain. a lllustrations showing the positions of the brains of trout (top) and goldfish (bottom). Unlike goldfish, the trout brain is located far below the head surface. b Sample coronal section stained to show Nissl material from a trout telencephalon (Trout B). The arrowheads depict the estimated recording locations. For reference, a mirror image outline of the brain section was drawn on the left. $\mathbf{c}$ All estimated locations for the three trout (color-coded dots) are depicted on the brain section outlines (Dm= pars medialis; $\mathrm{Dl}=$ pars lateralis, in the dorsal pallium). $\mathbf{d}$ Color-coded planes indicate the locations of the coronal brain sections where the recording locations were estimated for the three trout shown in $\mathbf{c}$

activity. Cells with $\leq 99$ spikes were excluded from the analysis. Cell type classification using the extracellular spike waveform feature was not performed, as the necessary criteria have not been established for fish neurobiology. The unit isolation quality was quantified for each cell based on the isolation distance index [14]. An example of the spike-sorting performance quality is shown in Fig. 5.

\section{Video tracking}

We used DeepLabCut ${ }^{\mathrm{TM}}$ (Mathis Lab, Cambridge, MA, USA) [15] to track the leading edge and fixed base of the 3D-printed neurologger case mounted on the skull through imagery captured at 30 frames per second. We used a USB 3.0 digital video camera with a non-distorting lens (C-Mount, Manual Iris, Wide Angle Lens, \# 89-524, Edmund Optics, Japan) mounted $1.5 \mathrm{~m}$ above the water tank (Fig. 1a); then, by concatenating the tracked fixed base of the case, we were able to reconstruct swimming trajectories (Fig. 7a). The image definition was set to $800 \times 800$ pixels. We computed the head direction from the tracked leading edge and fixed base using the inverse of the tangent function. 


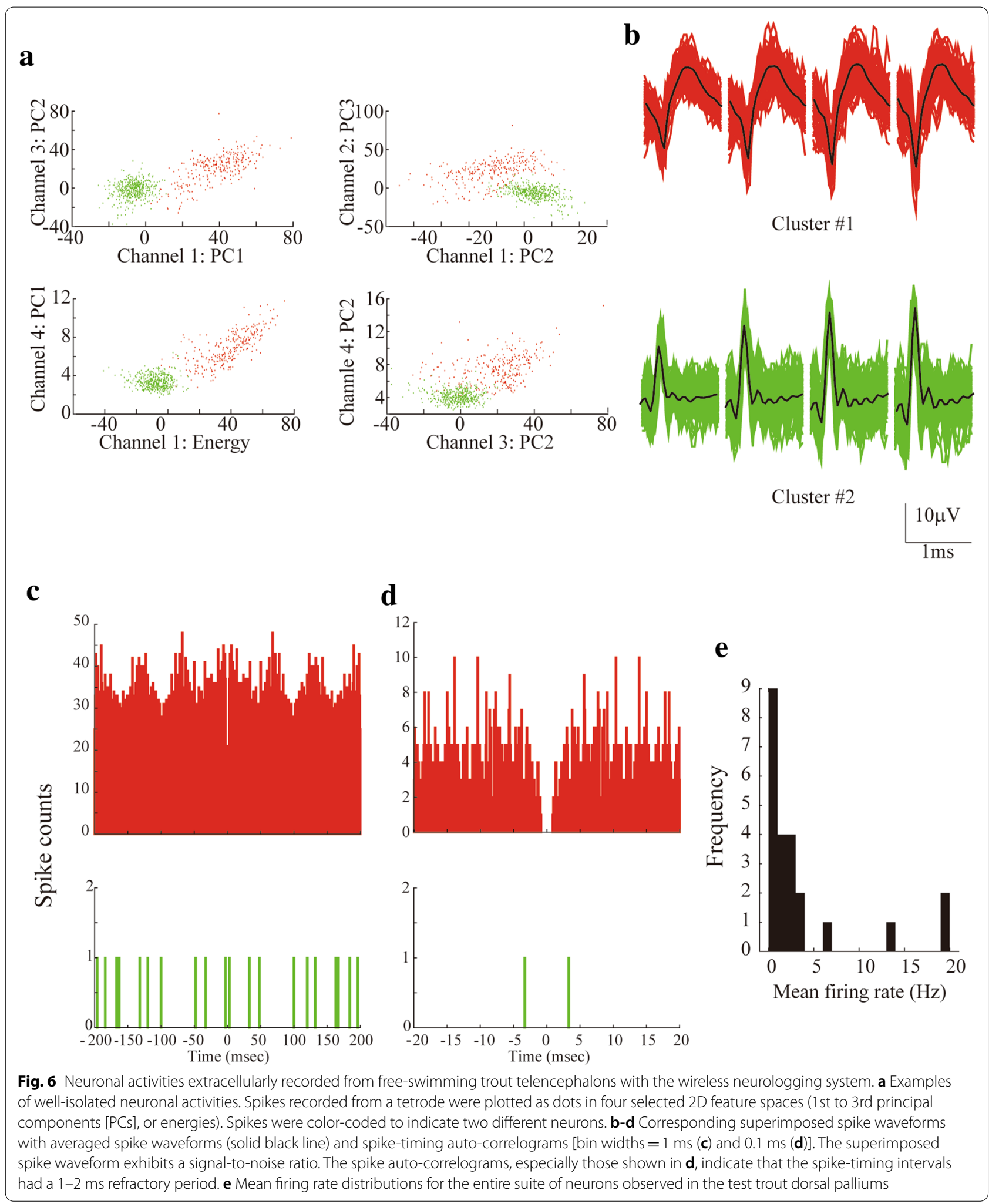




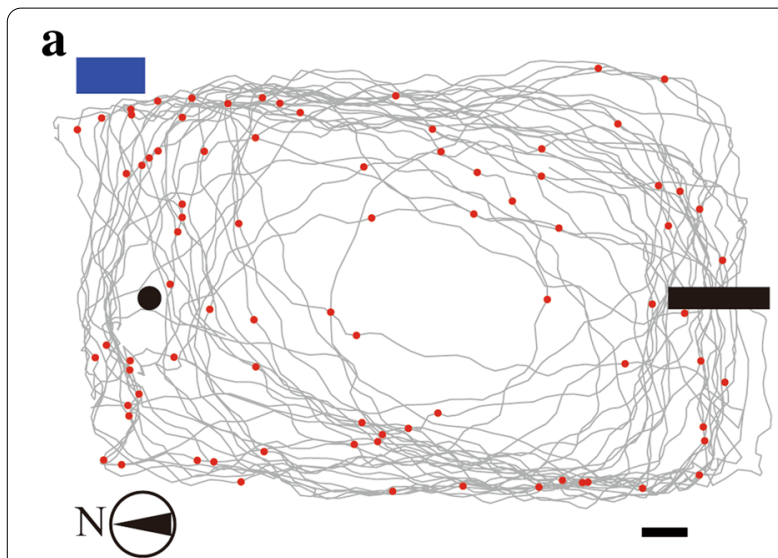

b
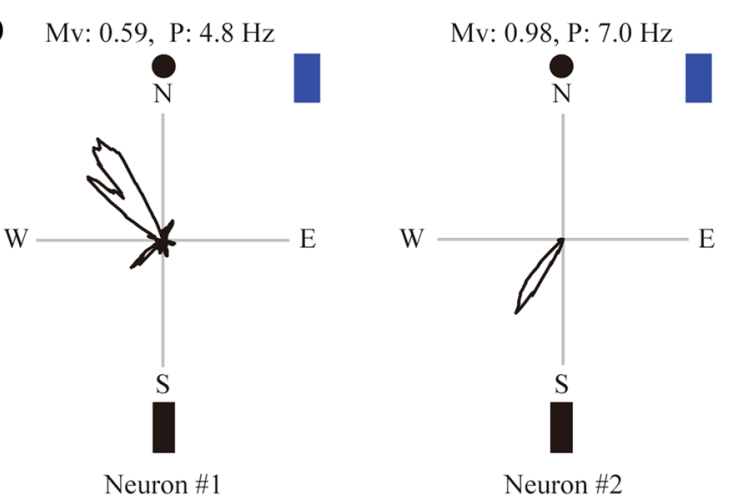

c
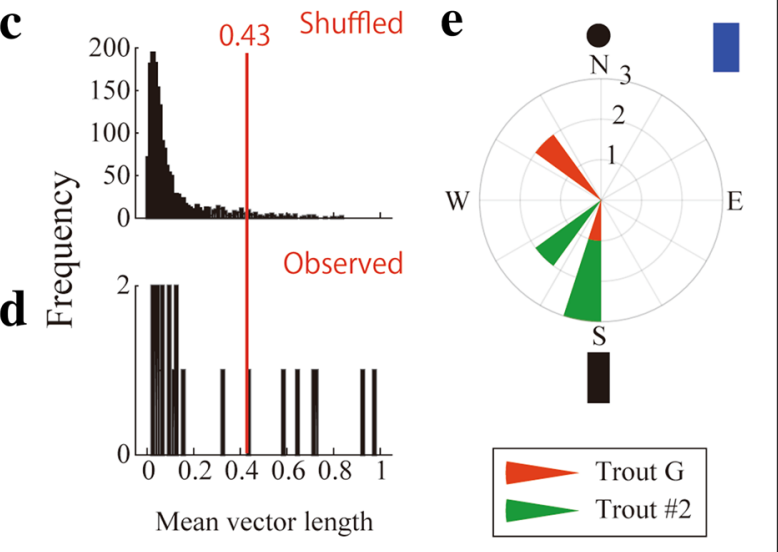

\section{Head direction cell analysis}

The directional tuning function for each cell was obtained by plotting its firing rate as a function of the fish head direction, divided into bins of $0.5^{\circ}$, and smoothed using a $14.5^{\circ}$ mean window filter.

The directional tuning strength was estimated by computing the mean vector length for the circular distribution of the firing rate. Head direction-modulated cells were defined as cells in the recorded data with mean vector lengths $>95$ th percentile of the shuffled data. For each permutation trial, the entire sequence of spikes fired by a
Fig. 7 Trout head direction cells. a Representative trajectory (gray line) with positions showing neuron-generated spikes (red dots) as recorded from the telencephalon of trout swimming in a water tank (scale bar $=10 \mathrm{~cm}$ ). Remarkable visible objects (pipe for water supply [black filled rectangle], hole for water sink [black filled circle], and radio transceiver [blue filled rectangle]), and compass bearing (bottom, left) are illustrated. b Polar plots of firing rate as a function of head direction, using examples from two representative neurons. The mean vector length $(\mathrm{Mv})$ and peak firing rate $(\mathrm{P})$ are indicated in the images. Visible objects are illustrated as in $\mathbf{a}$. $\mathbf{c}-\mathbf{d}$ Distribution of mean vector length for randomly shuffled data (c) and for the entire suite of neurons observed in our test trout dorsal palliums (d). The red line and the number indicate the 95th percentile for the shuffled data. e Circular distribution of the number of cells on the head orientation for seven individual head direction cells identified from trout $G$ and \#2. Head direction cells exist in the brains of trout $G$ (red) and \#2 (blue). In contrast, trout B had no head direction cells. Visible objects are illustrated as in $\mathbf{a}$

Table 1 Identified cells from the telencephalon of trout

\begin{tabular}{llll}
\hline Trout & G & \#2 & B \\
\hline \# of isolated cells & 3 & 6 & 14 \\
\# of head direction cells & 3 & 4 & 0 \\
\hline
\end{tabular}

cell was time-shifted along the fish swimming path with a random interval between $20 \mathrm{~s}$ and $20 \mathrm{~s}$ less than the trial length, with the end of the trial wrapped to the beginning. A head-direction-tuning function was then constructed, and the mean vector length was calculated. This procedure was repeated 100 times for each cell, the mean vector length distribution was computed for the entire set of permutations from all examined cells, and the $95^{\text {th }}$ percentile was determined.

\section{Analysis software}

All analyses were performed using custom-made programs based on MATLAB functions (v9.6; MathWorks, Natick, MA, USA).

\section{Histology}

The fish were deeply anesthetized with $0.5 \mathrm{~mL} / \mathrm{L}$ FA 100 and then transcardially perfused with $10 \%$ phosphatebuffered formalin fixative (3.5-3.8\% formaldehyde). The extracted brains were post-fixed overnight with Davidson's fixative (also known as Hartmann's fixative) solution (22.2 $\mathrm{mL} \mathrm{10 \%} \mathrm{buffered} \mathrm{formalin,} 32.0 \mathrm{~mL} 99 \%$ ethanol, $11.1 \mathrm{~mL}$ acetic acid, and $100 \mathrm{~mL}$ distilled water) at $4{ }^{\circ} \mathrm{C}$. The brains were incubated in gelatin solution (10\% gelatin in phosphate-buffered saline $[\mathrm{PBS}]$ ) at $37{ }^{\circ} \mathrm{C}$ for $4 \mathrm{~h}$ and solidified at $4{ }^{\circ} \mathrm{C}$. The brain embedded in the gelatin block was fixed in $10 \%$ phosphate-buffered formalin fixative (3.5-3.8\% formaldehyde) at $4{ }^{\circ} \mathrm{C}$ and sank in 
$30 \%$ sucrose in PBS at $4{ }^{\circ} \mathrm{C}$. The brains were cut coronally with a microtome (Ritratome REM-710, Yamato Koki Co., Saitama, Japan) set at $40 \mu \mathrm{m}$. The resulting brain slices were then stained with cresyl violet (SigmaAldrich, C5042-10G) to facilitate the examination of their cytoarchitecture.

\section{Results}

As a proof of principle, we recorded the activity of 23 cells from the telencephalons of three trout using a wireless biologging system with a lightweight neurologger enclosed in a 3D-printed, waterproof case. All recordings were made while the trout were voluntarily swimming in a rectangular water tank, and the fish did not exhibit reward-seeking behaviors during the recording periods. Nissl staining showed that the electrode tracks were distributed around the dorsal pallium of the telencephalon (Fig. 5). Previous fish studies have reported that the dorsal pallium is deeply involved in spatial learning and memory $[9,16-19]$.

\section{Neuronal activity recorded by the wireless logging system}

To confirm whether the signals recorded from the test trout brains originated from neurons, we examined spike statistics, including spike shape, firing rate, and spiketiming intervals. The recorded neurons typically exhibited a narrow spike shape [mean peak-to-trough spike width: $0.18 \pm 0.11 \mathrm{~ms}$ (mean \pm SD)]. However, cells showing a wider spike shape were also observed (widest spike width: $0.44 \mathrm{~ms}$ ). In the telencephalons of rodents, cells exhibiting a narrow spike shape and high firing rates are categorized as fast-spiking cells [20]. Although the necessary criteria have not been established for fish neurobiology, these results appeared to show that our biologging system could record different neuron types.

The mean firing rate distributions were skewed (median: $1.45 \mathrm{~Hz}$, Fig. 6e). The typical spike-timing autocorrelogram for identified single neurons demonstrated that the spike-timing intervals had a clear refractory period (1-2 ms) (Fig. 6d), suggesting that the identified neurons belonged physiologically to a single type. These results suggest that our system was capable of simultaneously recording multiple neuronal activities in the brains of free-swimming trout.

\section{Trout head direction cells}

Finally, to demonstrate our logging system capacity to examine neuronal correlation with fish movement, we examined the neuron firing rates as a function of fish swimming trajectory and heading (Fig. 7a). In some brain regions of rodents, including the anterior thalamus, subiculum, retrosplenial cortex, and entorhinal cortex, cells that maximally fire at a specific heading direction were found and called head direction cells [3]. Recently, a pioneering study also reported the presence of head direction cells in the telencephalon of goldfish [9]. Similarly, the neuronal activity of some cells in the trout telencephalons exhibited head direction preferences, becoming more active when their heads were oriented in a specific direction (Fig. 7b). Seven (30.4\%) of the 23 cells recorded passed the criterion to be classified as head direction cells (the mean vector length exceeded the 95th percentile of the mean vector lengths distribution in shuffled data). This number was significantly larger than expected for a random selection from the distribution $(P<0.001$, binomial test with an expected $P_{0}$ of 0.05$)$. Whereas cells in one trout did not exhibit head direction preferences, the head direction cells were found in the telencephalon of two of the three trout (Table 1). The heading orientations covered a wide span (Rayleigh's test for nonuniformity, $P>0.05, \mathrm{z}=-4.68$; Fig. $7 \mathrm{c}$ ), suggesting that the head direction preference was not due to artifacts. Overall, the head direction cells were tuned to neither a specific compass bearing nor visible objects, including pipes for water sink and source, and radio transceiver (Fig. 7e). However, at least one cell from trout $\mathrm{G}$ or \#2 mainly fired whenever the head pointed south or to the water supply. Furthermore, neighboring head direction cells recorded from the same electrode exhibited a similar heading trigger (trout G: two of three neighboring head direction cells; trout \#2: two of two neighboring head direction cells).

\section{Discussion}

We demonstrated the capacity of our novel wireless logging system to record neuronal activity in the brains of large salmonids. A previous pioneering study developed a wireless logging system for goldfish [8]; however, unlike goldfish, salmonids exhibit remarkable motherriver homing on a global scale. While swimming trajectories and accompanying external cues have been researched previously [21], neuronal activity in the brains of free-swimming salmonids has not been studied before because of the difficult access to the salmonid brain, located deep in the head, and the trout weight of several kilograms generating high torque. Thus, our system extends the wireless logging in goldfish, providing the possibility to examine the neuronal underpinning of underwater behavior in large salmonids.

In a previous study on the telencephalon of goldfish [9], we also found head direction cells in large salmonids. However, we could not investigate whether the head direction preference was tuned to specific cues, including geomagnetic compass bearing and landmarks, because of the lack of environmental manipulation. Further studies will be required to elucidate the neuronal underpinning of spatial information processing in large salmonids. 
Despite its exceptional potential in the field of animal biotelemetry, we could not test the ability to record neuronal activity in natural rivers where unpredictable events occur, such as higher water pressure, unexpected rocks, and animal recapture. Therefore, the question of whether our method can be used for mother-river homing remains unanswered. Technical improvements are required to address this question. For instance, the waterproof case square box shape must be formed with a streamlined shape against hydraulic resistance in rivers. Furthermore, since our system cannot synchronize external event signals in deeper water and only record neuronal activity for a few hours, neuronal recordings need to be operated automatically and locally above the fish's head. A state-of-the-art AI-assisted biologger [22] can accomplish such tasks, detect interest behaviors in real time, and activate sensors such as gyroscopes, acceleration, and water depth. In a natural environment, it is desirable to run this system with an AI-assisted biologger to start recording neuronal activity at the time of interest.

\section{Conclusions}

In the present study, we developed a wireless logging system capable of recording multiple neuronal activities from the brains of free-swimming trout, a large salmonid. The spike-timing intervals of the recorded neurons with a specific refractory period suggested that the system could precisely record multiple neuronal activities. Using the system on trout swimming in a water tank, we found head direction cells in their telencephalons, firing maximally in specific head directions, suggesting that the system has the potential to examine the space-responsive properties of neurons in fish brains. We anticipate that our system will stimulate the process of examining spatial cognition mechanisms in salmonid brains.

\section{Abbreviations}

EIB: Electrode interface board; PBS: Phosphate-buffered saline.

\section{Acknowledgments}

We thank Kazuma Hase and Yojiro Yokomori for their helpful support in the surgical preparations.

\section{Authors' contributions}

$\mathrm{ST}, \mathrm{KY}, \mathrm{TK}$, and YM conceived the project, while KI, SO, and ST performed the histological verification. KI made tetrodes and waterproof cases designed by $\mathrm{ST}$, and $\mathrm{ST}, \mathrm{YM}, \mathrm{TH}$, and RT performed the electrophysiological experiments and surgery. ST performed data analyses, and ST and YM wrote the manuscript, with inputs from all other authors. All authors read and approved the final manuscript.

\section{Funding}

This work was supported by the JSPS KAKENHI (Grant numbers $16 \mathrm{H06543}$ and $19 \mathrm{H} 01131$ to S.T., $15 \mathrm{~K} 07229$ to Y.M., and $16 \mathrm{H} 06541$ to K.Y.).

\section{Availability of data and materials}

The 3D CAD designs for the waterproof case and tetrode housing are available at https://github.com/TakahashiLab/Trout. The datasets used and/or analyzed during the current study are available from the corresponding author upon reasonable request.

\section{Ethics approval and consent to participate}

All procedures were approved by the University of Tokyo Institutional Animal Care and Use Committee (\#A-19-8).

\section{Consent for publication}

Not applicable.

\section{Competing interests}

The authors declare no competing interests.

\section{Author details}

${ }^{1}$ Laboratory of Cognitive and Behavioral Neuroscience, Graduate School of Brain Science, Doshisha University, Kyotanabe City, Kyoto 610-0394, Japan. ${ }^{2}$ Graduate School of Environmental Studies, Nagoya University, Furo-cho, Chikusa-ku, Nagoya, Aichi 464-8601, Japan. ${ }^{3}$ Department of Cell Biology and Neuroscience, Graduate School of Medicine, Juntendo University, Bunkyo-ku, Tokyo 113-8421, Japan. ${ }^{4}$ International Coastal Research Center, The Atmosphere and Ocean Research Institute, The University of Tokyo, 1-19-8, Akahama, Otsuchi, Iwate 028-1102, Japan. ${ }^{5}$ College of Bioresource Sciences, Nihon University, Kanagawa 252-0813, Japan.

Received: 19 October 2020 Accepted: 27 January 2021

Published online: 12 February 2021

\section{References}

1. O'Keefe J, Nadel L. The hippocampus as a cognitive map. New York: Oxford University Press; 1978.

2. Hafting T, Fyhn M, Molden S, Moser M, Moser El. Microstructure of a spatial map in the entorhinal cortex. Nature. 2005;436(7052):801-6.

3. Taube JS, Muller RU, Ranck JB. Head-direction cells recorded from the postsubiculum in freely moving rats. I. Description and quantitative analysis. J Neurosci. 1990;10:420-35.

4. Buzsáki G. Neuroscience: neurons and navigation. Nature. 2005;436(7052):781-2.

5. Kim DH, Kim J, Marques JC, Grama A, Hildebrand DGC, Gu W, et al. Panneuronal calcium imaging with cellular resolution in freely swimming zebrafish. Nat Methods. 2017;14(11):1107-14.

6. Canfield JG, Mizumori SJY. Methods for chronic neural recording in the telencephalon of freely behaving fish. J Neurosci Methods. 2004;133(1-2):127-34.

7. Yartsev MM, Ulanovsky N. Representation of three-dimensional space in the hippocampus of flying bats. Science. 2013;340(6130):367-72.

8. Vinepinsky E, Donchin O, Segev R. Wireless electrophysiology of the brain of freely swimming goldfish. J Neurosci Methods. 2017;278:76-86.

9. Vinepinsky E, Cohen L, Perchik S, Ben-Shahar O, Donchin O, Segev R. Representation of edges, head direction, and swimming kinematics in the brain of freely_navigating fish. Sci Rep. 2020;10(1):1-16.

10. Takahashi S. Hierarchical organization of context in the hippocampal episodic code. Elife. 2013;2013(2):e00321.

11. Takahashi S. Episodic-like memory trace in awake replay of hippocampal place cell activity sequences. Elife. 2015. https://doi.org/10.7554/eLife 08105 .

12. Gray CM, Maldonado PE, Wilson M, McNaughton B. Tetrodes markedly improve the reliability and yield of multiple single-unit isolation from multi-unit recordings in cat striate cortex. J Neurosci Methods. 1995;63(1-2):43-54

13. Harris KD, Henze DA, Csicsvari J, Hirase H, Buzsaki G. Accuracy of tetrode spike separation as determined by simultaneous intracellular and extracellular measurements. J Neurophysiol. 2000;84(1):401-14.

14. Harris KD, Hirase H, Leinekugel X, Henze DA, Buzsaki G, Buzsáki G. Temporal interaction between single spikes and complex spike bursts in hippocampal pyramidal cells. Neuron. 2001;32(1):141-9. 
15. Mathis A, Mamidanna P, Cury KM, Abe T, Murthy VN, Mathis MW, et al. DeepLabCut: markerless pose estimation of user-defined body parts with deep learning. Nat Neurosci. 2018;21(9):1281-9.

16. Saito K, Watanabe S. Spatial learning deficits after the development of dorsomedial telencephalon lesions in goldfish. Neuroreport. 2004;

17. Saito K, Watanabe S. Deficits in acquisition of spatial learning after dorsomedial telencephalon lesions in goldfish. Behav Brain Res. 2006;172(2):187-94

18. Rodríguez F, López JC, Vargas JP, Gómez Y, Broglio C, Salas C. Conservation of spatial memory function in the pallial forebrain of reptiles and ray-finned fishes. J Neurosci. 2002;22(7):2894-903.

19. Salas C, Broglio C, Rodríguez F. Evolution of forebrain and spatial cognition in vertebrates: conservation across diversity. Brain Behav Evol. 2003;62(2):82-92

20. Bartho P, Hirase H, Monconduit L, Zugaro M, Harris KD, Buzsaki G. Characterization of neocortical principal cells and interneurons by network interactions and extracellular features. J Neurophysiol. 2004;92(1):600-8. https://doi.org/10.1152/jn.01170.2003.

21. Putman NF, Lohmann KJ, Putman EM, Quinn TP, Klimley AP, Noakes DLG. Evidence for geomagnetic imprinting as a homing mechanism in pacific salmon. Curr Biol. 2013;23(4):312-6.

22. Korpela J, Suzuki H, Matsumoto S, Mizutani Y, Samejima M, Maekawa T, et al. Machine learning enables improved runtime and precision for biologgers on seabirds. Commun Biol. 2020. https://doi.org/10.1038/s4200 3-020-01356-8.

\section{Publisher's Note}

Springer Nature remains neutral with regard to jurisdictional claims in published maps and institutional affiliations.
Ready to submit your research? Choose BMC and benefit from:

- fast, convenient online submission

- thorough peer review by experienced researchers in your field

- rapid publication on acceptance

- support for research data, including large and complex data types

- gold Open Access which fosters wider collaboration and increased citations

- maximum visibility for your research: over 100M website views per year

At $\mathrm{BMC}$, research is always in progress.

Learn more biomedcentral.com/submissions 